Ecology

\title{
Chalk-hill blues
}

From the southern

Mediterranean to the northern half of Europe, the distinctive colour of the chalk-hill blue butterfly Polyommatus coridon (pictured) is unmissable - in contrast to the species itself, which has a rather patchy distribution. In the context of general concern over the effect that changes in climate and land use might have on biodiversity, T. Schmitt and G. M. Hewitt used this species, and another Erebia medusa, the woodland ringlet - to test ideas about a species' history, its present-day patterns of genetic diversity and its robustness.

As they report in Molecular Ecology (13, 21-31; 2004), Schmitt and Hewitt have drawn on various datasets to conclude that the butterflies' location in Europe, determined by past climatic events, reflects their genetic diversity, and also, they speculate, their predisposition to population decline.

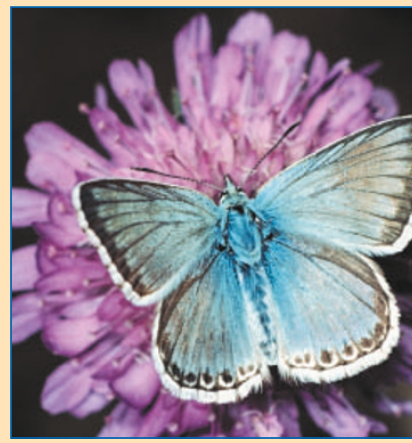

There are two major lineages of the chalk-hill blue in Europe, one in the east, the other in the west. After the last ice age, these groups probably emerged from two regions in the southern Mediterranean, and then underwent a northward postglacial colonization. Although the two lineages show equivalent genetic diversity, when the authors compared northern and southern populations of each, those further north were considered genetically poor, with respect to diversity, and less stable demographically, than their southern counterparts. A similar pattern emerged for the woodland ringlet, only with a west-east axis.

So for both the woodland ringlets and the chalk-hill blues, the least stable populations - and so those with apparently less adaptability to change - are those furthest away from their former refugial areas. On the face of it, then, the butterflies ability to adapt is a correlate of genetic diversity. If matters are to be taken further in examining the relationship between 'phylogeographical' patterns and population stability in European butterflies, more detailed genetic surveys will be required of these as well as other species - in addition to an assessment of the relative importance of genetic constitution and ecological change.

Katrin Bussel temperature superconductors is the link between their layered crystal structure and their transition temperatures (at which they become superconducting). These ceramic materials contain multiple layers of superconducting copper oxide, separated by insulating material - a kind of superconducting baklava. As physicists learned to synthesize more complex versions of these materials, with first one, then two, then three superconducting layers, the superconducting transition temperature shot up, from about $40 \mathrm{~K}$ in the single-layer materials to above $130 \mathrm{~K}$ in the three-layer materials ${ }^{1,2}$ (Fig. 1). But there it has stood for almost a decade — adding more layers unfortunately drives the transition temperature back down again. So what went wrong?

On page 53 of this issue, Chakravarty, Kee and Völker ${ }^{3}$ propose an explanation. Their theory brings together several insights into the physics of high-temperature superconductivity, and links three important effects — tunnelling, interlayer charge distribution and hidden order.

The first element of Chakravarty and colleagues' analysis ${ }^{3}$ is electron tunnelling between the superconducting layers. Inside a superconductor, electrons are bound into pairs. When two superconducting layers are brought close together, these pairs can 'tunnel' or jump from one to the other. (Here 'tunnelling' refers to the quantum-mechanical motion of a wave/particle through a region of space that would be forbidden in classical mechanics.) This tunnelling effect couples the two superconductors, and is called a 'Josephson coupling' after its discoverer.

About ten years ago (in a now disproven theory), Chakravarty, Sudbø, Anderson and Strong proposed that interlayer tunnelling is actually the origin of high-temperature superconductivity ${ }^{4}$. Chakravarty et al. ${ }^{3}$ have revived the idea of interlayer coupling, but now they argue that, although it is not the main engine of superconductivity in the layers, such coupling is responsible for the rise in transition temperature when going from one-layer to three-layer materials.

The authors ${ }^{3}$ also take account of how charge is distributed between the layers of the superconductor. A remarkable aspect of hightemperature superconductors is that, in many ways, these materials are closer to insulators than to metals. The mother compounds of high-temperature superconductors are insulating, and develop superconductivity only when extra charge carriers (electrons, or their positively charged counterparts, holes) are introduced into the material by chemical doping. Some years ago, Japanese physicists Ohta, Tohyama and Maekawa ${ }^{5}$ predicted that in multilayer high-temperature materials the charge would redistribute, with the result that doping in the interior layers would in fact be less than in the outer layers. This idea has been confirmed by NMR measurements ${ }^{6,7}$ of the doping profile across the multilayer.

But central to the concepts underlying the new theory is the so-called order parameter. The Russian physicist Lev Landau was the first to realize that when matter develops new forms of order, the collective motion of its constituent particles can be described by variables that he called 'order parameters'. The advantage of Landau's order-parameter approach is that it means that the collective large-scale properties of a system can be easily separated from the gothic details of its microscopic motions. Using this concept, Landau and Vitaly Ginzburg ${ }^{8}$ were able to develop an order-parameter theory for the macroscopic properties of superconductors more than a decade before the pairing mechanism for superconductivity was devised by John Bardeen, Leon Cooper and Robert Schrieffer.

High-temperature superconductors are far more complex than their low-temperature counterparts, and there are many indications that their unique properties result from the competition between more than one type of order parameter. The electron correlations that are responsible for high-temperature superconductivity are still a mystery. But Chakravarty et al. ${ }^{3}$ bypass this unsolved problem by using the order-parameter approach to analyse the interaction between the superconductivity in each layer of the material.

The authors have melded these ideas of tunnelling, charge transfer and order into a simple Landau-Ginzburg expression for the total energy of the system. Looking beyond the order connected with correlated electrons, the basic idea of their theory is that, for more than three layers of copper oxide, a second, competing order parameter nucleates in the interior under-doped layers and lowers the superconducting transition temperature. By fitting the properties of a singlelayer material and using the measured doping profile, they are then able to compute how the superconducting transition temperature evolves with the number of layers, obtaining good qualitative agreement with experimental data.

A key prediction of Chakravarty and colleagues' theory ${ }^{3}$ is that the 'pseudogap' should be larger in the inner layers of a multilayer superconductor. In normal superconductors, when electrons pair up, a gap develops in the electron energy spectrum; the higher the transition temperature of the metal, the larger the gap. But high-temperature superconductors break this rule: under-doped materials with lower transition temperatures actually develop a larger gap than optimally doped materials, even at high temperatures where they have not yet become superconducting. The nature of this so-called pseudogap is a 